\title{
COMPARATIVE STUDY OF DIFFERENT FORECASTING METHODS FOR VARIATION IN GOLD PRICE
}

\author{
Rajul Namdeo \\ Department of Mechanical Engineering, \\ Babulal Tarabai Institute of Research \& \\ Technology, Sagar (M.P.), India
}

\begin{abstract}
In this result paper, we used four different forecasting techniques i.e. moving average, weighted moving average, exponential smoothing and regression analysis method. Now, these forecasting techniques compared with each other and find the suitable forecast technique to provide accurate gold price near about original gold price in market. After calculation, we find that exponential smoothing method provide better forecasting result for gold price and these gold price help the investors to know the suitable time for buy and selling the gold.
\end{abstract}

Keywords - Forecasting, Moving Average, Weighted Moving Average, Exponential Smoothing, Regression Analysis, Gold Price.

\section{INTRODUCTION}

Forecasting is defined as, "The process of finding a future demand by using past data or past experience."

According to Evan J. Douglas, "Demand forecasting may be defined as the process of finding values for demand in future time periods [3]

Various application of forecasting is as follows:-

- Supply Chain Management

- Economic forecasting

- Earthquake forecasting

- Technology forecasting

- Weather forecasting, etc.

\section{EFFORTS BY VARIOUS RESEARCHERS}

[1] Syed Misbah Uddin, Aminur Rahman, Emtiaz Uddin Ansari (2017), "comparison of some statistical forecasting techniques with GMDH predictor: a case study", the aim of this research to determine the accurate models for forecasting the cement demand. In this research monthly sales data of cement from ranging Jan 2007 to Feb 2016 is used and also Group Method of Data Handling (GMDH) model used to forecasting the cement demand. The time series smoothing techniques such as exponential smoothing, double exponential

\author{
Tarun Kumar Yadav \\ Department of Mechanical Engineering, \\ Babulal Tarabai Institute of Research \& \\ Technology, Sagar (M.P.), India
}

smoothing, moving average, weightage moving average and regression method were also used in this research.

Then, the original data were compared to the forecast produced by the time series model and GMDH model and for comparing the forecasting accuracy mean absolute deviation (MAD), mean absolute percentage error (MAPE) and mean square error (MSE) were also calculated. These comparison shows that the GMDH provide better result than other statistical models based on MAD, MAPE and MSE.

[2] Alessio Azztti (2016), "Forecasting Gold Price: A Comparative Study", this research evaluates the correctness of various existing forecasting techniques and models to provide accurate gold prices forecast. It was found that ARIMA model provided the best forecast of prices over 36 month forecasting horizons as compared to other models. ARIMA model provided better result to metal such as silver, platinum, palladium and rhodium as well.

\section{METHODOLOGY}

A. Moving Average Method - Moving average may be defined as the average of a fixed number of items in the series, which move through the series by dropping the top item of the previous averaged group and adding the next item below in each successive average. Adding all the value for a certain number of successive periods and then dividing the sum obtained by the number of items include compute moving average.[5]

B. Weighted Moving Average - Weighted moving average method allows any weights to be placed on each element of the data, provide that sum of all weights equals 1.[4]

The general, forecasting for the $\mathrm{t}^{\mathrm{th}}$ period is given by,

$$
\mathrm{F}_{\mathrm{t}}=\mathrm{w}_{1} \times \mathrm{D}_{\mathrm{t}-1}+\mathrm{w}_{2} \times \mathrm{D}_{\mathrm{t}-2}+\cdots+\mathrm{w}_{\mathrm{n}} \times \mathrm{D}_{\mathrm{t}}
$$
Where

$\mathrm{W}_{1}, \mathrm{w}_{2}, \ldots, \mathrm{W}_{\mathrm{n}}=$ weights given to the actual data for periods $\mathrm{t}-1, \mathrm{t}-2, \ldots, \mathrm{t}-\mathrm{n}$ respectively.

$D_{1}, D_{2} \ldots \ldots . . D_{t-n}=$ actual data for periods $t-1, t-2, \ldots \ldots$, $\mathrm{t}-\mathrm{n}$ respectively. 


\section{International Journal of Engineering Applied Sciences and Technology, 2019 \\ Vol. 4, Issue 5, ISSN No. 2455-2143, Pages 210-213 \\ Published Online September 2019 in IJEAST (http://www.ijeast.com)}

C. Exponential Smoothing Method - It is based on the forecast and the actual demand for the previous period and a smoothing constant $\alpha$ which lies between 0 and 1. [4]

According to this method forecast for the $t_{\text {th }}$ period is given by the relation,

$$
\begin{aligned}
\mathrm{F}_{\mathrm{t}} & =\mathrm{F}_{\mathrm{t}-1}+\alpha\left(\mathrm{D}_{\mathrm{t}-1}-\mathrm{F}_{\mathrm{t}-1}\right) \\
& =\alpha \times \mathrm{D}_{\mathrm{t}-1}+(1-\alpha) \times \mathrm{F}_{\mathrm{t}-1}
\end{aligned}
$$

D. Regression Analysis - Regression can be defined as a functional relationship between two or more correlated variables. One variable is known or assumed and used to forecast the value of un-known variable. The relationship is usually developed from the observed data. The relationship between variables appears to be linear the regression is called linear regression.[4]

The linear regression line is of the

Where,

$$
\mathrm{Y}=\mathrm{a}+\mathrm{bX}
$$

\footnotetext{
$\mathrm{Y}=$ Dependent variable, which is need to be forecast

$\mathrm{X}=$ Independent variable

$\mathrm{a}=$ Intercept on $\mathrm{y}$-axis

$\mathrm{b}=$ Slope of the line
}

\section{DATA COLLECTION}

Data collection of previous $8^{\text {th }}$ months is shown in Appendix A.

\section{RESULT TABLE}

\begin{tabular}{|c|c|c|c|c|c|}
\hline \multicolumn{7}{|c|}{ Gold Price Forecasting For June 2019 } \\
\hline Date & $\begin{array}{c}\text { Original } \\
\text { Gold } \\
\text { Price }\end{array}$ & $\begin{array}{c}\text { Moving } \\
\text { Average }\end{array}$ & $\begin{array}{c}\text { Weighted } \\
\text { Moving } \\
\text { Average }\end{array}$ & $\begin{array}{c}\text { Exponential } \\
\text { Smoothing }\end{array}$ & $\begin{array}{c}\text { Regression } \\
\text { Analysis }\end{array}$ \\
\hline 1-Jun-2019 & 32975 & 32758.33 & 32752.5 & 32719.17 & 32717.86 \\
\hline 2-Jun-2019 & 32975 & 32833.33 & 32865 & 33853.33 & 32889.29 \\
\hline 3-Jun-2019 & 33200 & 32891.67 & 32925 & 32994.17 & 33032.14 \\
\hline 4-Jun-2019 & 33250 & 33050 & 33087.5 & 33202.5 & 32689.29 \\
\hline 5-Jun-2019 & 33550 & 33141.67 & 33180 & 33263.33 & 32982.14 \\
\hline 6-Jun-2019 & 33600 & 33333.33 & 33390 & 33509.17 & 33289.29 \\
\hline 7-Jun-2019 & 33750 & 33466.67 & 33515 & 33573.33 & 33596.43 \\
\hline 8-Jun-2019 & 33725 & 33633.33 & 33665 & 33721.67 & 33889.29 \\
\hline 9-Jun-2019 & 33725 & 33691.67 & 33707.5 & 33715.83 & 34107.14 \\
\hline 10-Jun-2019 & 33450 & 33733.33 & 33730 & 33721.67 & 34217.86 \\
\hline 11-Jun-2019 & 33350 & 33633.33 & 33587.5 & 33478.33 & 33732.14 \\
\hline 12-Jun-2019 & 33500 & 33508.33 & 33455 & 33378.33 & 33460.71 \\
\hline 13-Jun-2019 & 33650 & 33433.33 & 33445 & 33500.83 & 33389.29 \\
\hline 14-Jun-2019 & 33800 & 33500 & 33545 & 33628.33 & 33432.14 \\
\hline 15-Jun-2019 & 33850 & 33650 & 33695 & 33770 & 33617.86 \\
\hline
\end{tabular}

VI. RESULT GRAPH

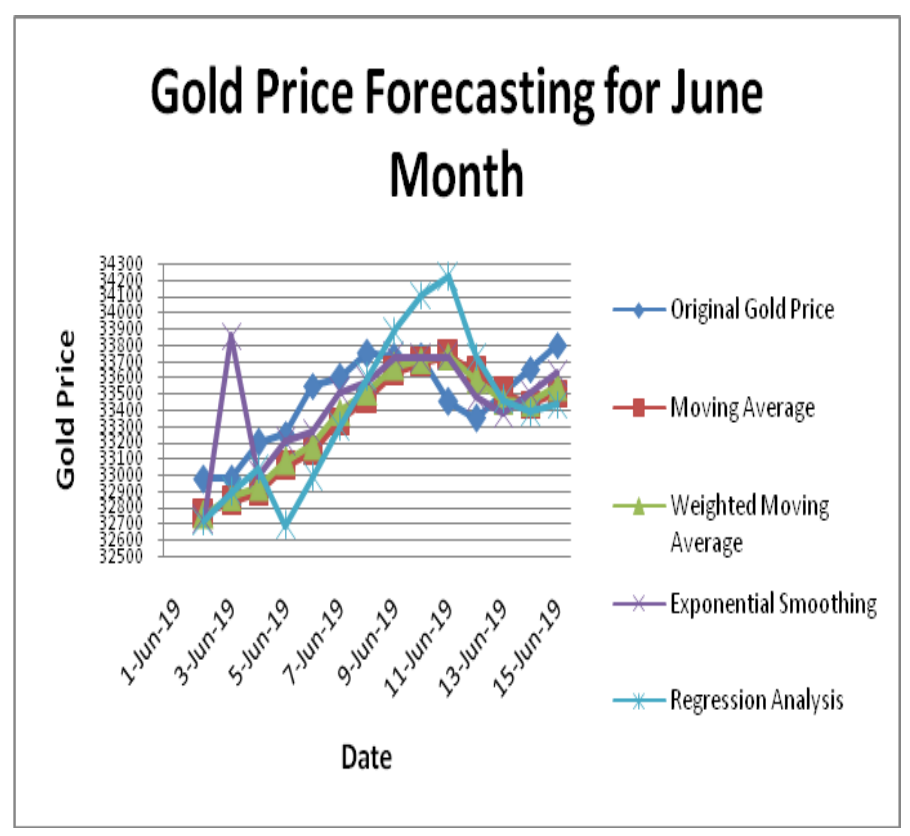

\section{CONCLUSION}

The aim of this research to forecast the gold price and also compare the different types of forecasting techniques i.e. moving average, weighted moving average, exponential smoothing and regression analysis method. For this purpose, four different types of forecasting techniques were used and these forecasting techniques compare with each other. Then, we find the best suitable forecasting method for gold price. After observation we find that, exponential smoothing method gives the better gold price forecasting.

\section{ACKNOWLEDGEMENT}

It gives me immense pleasure to express my deepest sense of gratitude and sincere thanks to my highly respected and esteemed guide Mr. Tarun Kumar Yadav, MECHANICAL ENGINEERING DEPARTMENT, BTIRT, Sagar for their valuable guidance, encouragement and help for completing this work. Their useful suggestions for this whole work and cooperative behavior are sincerely acknowledged.

I would like to express my sincere thank to Mr. Akash Tomar, MECHANICAL ENGINEERING DEPARTMENT, BTIRT, Sagar for giving me this opportunity to undertake this Research.

I also wish to express my indebtedness to my parents as well as my family member whose blessings and support always helped me to face the challenges ahead. 


\section{International Journal of Engineering Applied Sciences and Technology, 2019 \\ Vol. 4, Issue 5, ISSN No. 2455-2143, Pages 210-213 \\ Published Online September 2019 in IJEAST (http://www.ijeast.com)}

\section{REFERENCE}

[1] Uddin Syed Misbah, Rahman Aminur, Emtiaz Uddin Ansari. (Dec 2017), "Comparison of Some Statistical Forecasting Techniques with GMDH Predictor: A Case Study", Journal of Mechanical Engineering, Vol. ME 47

[2] Azztti Alessio (27 Feb 2016), "Forecasting Gold Price: A Comparative Study".

[3] https://brainly.in/question/1618623, Time: 8.15 pm, Date: 20-11-2018

[4] Chase Richard B., Shankar Ravi, Jacobs F. Robert , "Operations and Supply Chain Management", Fourteen Edition, Page No. 553

[5] Murthy P. Rama, "Production And Operations Management", Revised Second Edition, Page No. 37

[6] Abidin Syazwani Zainal et al. (2018), "Comparison on Estimating Malaysia Gold Price via Nonlinear Prediction Method and Box-Jenkins Model",.

[7] Dehghani Hesam and Zangeneh Mahsa (23 July 2018), "Crude Oil Price Forecasting: A Biogeography-Based
Optimization Approach", ISSN: 1556-7249 (Print) 15567257 (Online)

[8] Tripathy Naliniprava (2017), "Forecasting Gold Price with Auto Regressive Integrated Moving Average Model”, ISSN: 2146-4138, IJEFI, 2017, 7(4), 324-329.

[9] Cenas Paulo V. (Nov 2017), "Forecast of Agricultural Crop Price using Time Series and Kalman Filter Method", Asia Pacific Journal of Multidisciplinary Research Vol. 5 No.4, 1521 November 2017 Part III P-ISSN 2350-7756 E-ISSN 2350-844.

[10] Guha Banhi Guha and Bandyopadhyay Gautam Bandyopadhyay (March 2016), "Gold Price Forecasting Using ARIMA Model", Journal of Advanced Management Science Vol. 4, No. 2, pp. 117-121 P. Tay and J. Havlicek, "Image Watermarking Using Wavelets", in Proceedings of the 2002 IEEE, pp. II.258 - II.261, 2002.

\section{APPENDIX - A. Previous $8^{\text {th }}$ Month Gold Rate}




\begin{tabular}{|c|c|c|c|c|c|c|c|c|}
\hline Date & $\begin{array}{l}\text { Oct. } \\
\text { (2018) }\end{array}$ & $\begin{array}{l}\text { Nov. } \\
\text { (2018) }\end{array}$ & $\begin{array}{c}\text { Dec } \\
(2018)\end{array}$ & $\underset{(2019)}{\text { Jan }}$ & $\begin{array}{c}\text { Feb } \\
(2019)\end{array}$ & $\begin{array}{l}\text { March } \\
\text { (2019) }\end{array}$ & $\begin{array}{c}\text { April } \\
\text { (2019) }\end{array}$ & $\begin{array}{c}\text { May } \\
\text { (2019) }\end{array}$ \\
\hline 1 & 30601 & 32350 & 31160 & 32325 & 33950 & 33450 & 32425 & 32750 \\
\hline 2 & 30589 & 32100 & 31160 & 32500 & 33990 & 33425 & 32625 & 32300 \\
\hline 3 & 31036 & 32100 & 31750 & 32575 & 33990 & 33425 & 32425 & 32425 \\
\hline 4 & 31154 & 32125 & 31725 & 32300 & 33960 & 33375 & 32550 & 32475 \\
\hline 5 & 31154 & 32400 & 31825 & 32350 & 33950 & 32900 & 32650 & 32475 \\
\hline 6 & 31645 & 32125 & 31850 & 32350 & 33925 & 32550 & 32650 & 32550 \\
\hline 7 & 31645 & 32130 & 32050 & 32525 & 33900 & 32525 & 32650 & 32575 \\
\hline 8 & 31649 & 32150 & 32300 & 32575 & 33925 & 32975 & 32900 & 32800 \\
\hline 9 & 31380 & 32125 & 32300 & 32825 & 33910 & 33050 & 32900 & 32950 \\
\hline 10 & 31381 & 32175 & 32875 & 32800 & 33910 & 33050 & 32925 & 32875 \\
\hline 11 & 31997 & 32175 & 32825 & 32850 & 33800 & 32650 & 32800 & 32950 \\
\hline 12 & 31816 & 31950 & 32600 & 32825 & 33450 & 32700 & 32740 & 32950 \\
\hline 13 & 31842 & 31800 & 32575 & 32825 & 33675 & 33100 & 32425 & 33250 \\
\hline 14 & 31842 & 31825 & 32500 & 33200 & 33700 & 32750 & 32700 & 33400 \\
\hline 15 & 32278 & 31650 & 32475 & 33250 & 33975 & 32800 & 32680 & 33250 \\
\hline 16 & 32152 & 31625 & 32475 & 33375 & 33950 & 32825 & 32675 & 33150 \\
\hline 17 & 31990 & 31625 & 32300 & 33350 & 33950 & 32825 & 32575 & 33050 \\
\hline 18 & 32089 & 32400 & 32100 & 33100 & 34100 & 32475 & 32525 & 33925 \\
\hline 19 & 32084 & 31450 & 31975 & 33050 & 34225 & 32900 & 32450 & 32900 \\
\hline 20 & 31910 & 31425 & 32175 & 33050 & 34500 & 32850 & 32500 & 32650 \\
\hline 21 & 31910 & 31375 & 32250 & 33000 & 34000 & 32925 & 32500 & 32600 \\
\hline 22 & 31914 & 31350 & 32050 & 33125 & 34050 & 33050 & 32650 & 32650 \\
\hline 23 & 32271 & 31600 & 32050 & 33150 & 33975 & 32950 & 32600 & 32700 \\
\hline 24 & 32066 & 31300 & 32225 & 33000 & 33975 & 32950 & 32780 & 32625 \\
\hline 25 & 32095 & 31150 & 32325 & 33225 & 33850 & 32975 & 32850 & 32675 \\
\hline 26 & 32156 & 31150 & 32275 & 33425 & 33875 & 32900 & 32950 & 32675 \\
\hline 27 & 31932 & 31225 & 32450 & 33425 & 33775 & 32600 & 32950 & 32600 \\
\hline 28 & 31932 & 31100 & 32600 & 33500 & 33550 & 32775 & 32930 & 32450 \\
\hline 29 & 32012 & 31225 & 32525 & 33650 & - & 32800 & 32950 & 32750 \\
\hline 30 & 31926 & 31050 & 32525 & 33725 & - & 32750 & 32850 & 32800 \\
\hline 31 & 31886 & - & 32375 & 33900 & - & 32750 & - & 32725 \\
\hline
\end{tabular}

